\title{
Continous idiopathic facial myokymia succesfully treated with botulinum toxin
}

\author{
Abstract \\ Introduction: Botulinum toxin infiltration has been suggested useful for focalized \\ myokymia. \\ Methods: 71-year old woman presented continuous undulating movements in her right \\ cheek. EMG was consistent with myokymia. \\ Results: Five units of Onabotulinumtoxin A abolished myokymia. \\ Conclusion: Low doses of Onabotulinumtoxin A are useful in continuous idiopathic facial \\ myokymia.
}

Keywords: Myokymia; Botulinum Toxin; Treatment
Volume 6 Issue 3 - 2017

\author{
Carol Aguilar Alvarado, Gonzalo Castro, \\ Federico Micheli \\ University of Buenos Aires, Argentina
}

\begin{abstract}
Correspondence: Federico Micheli, MD PhD, Parkinson's Disease and Movement Disorders Unit. Hospital de Clínicas "José de San Martín”. University of Buenos Aires, Juncal 1695 Piso 5] Zip code 162, Buenos Aires, Argentina, Tel 54 I I-48 I I3076, Fax 54 I I-48 I I-3076, Email fmicheli@fibertel.com.ar
\end{abstract}

Received: November 18, 2016 | Published: March 02, 2017

\section{Letter to editor}

Facial myokymia are subtle involuntary undulating or vermicular muscular contractions that spread throughout the striated muscles supplied by the facial nerve. ${ }^{1}$ Simultaneous or sequential triggers activate one or multiple motor units, and usually manifest as spontaneous muscular activity with brief repetitive discharges of action potentials that repeat rhythmically or semi-rhythmically. Short intervals of irregular electrical silence between 100 and 200 milliseconds (and up to 3 seconds) occur before the next myokymia discharge. ${ }^{2}$

The pathogenesis is still unclear. Given the association of myokymia with a variety of lesion sites, its origin seems to occur at any level of the motor pathway from the motor neurons to the distal segments of the nerve terminal. Facial myokymia is believed to originate in the facial motor nucleus either by direct irritation, hyper-excitation, hyper-sensitivity to secondary denervation of a supranuclear process or motor-neural isolation of the facial nucleus of the internuncial neuron pool that mediates the sensory afference. ${ }^{3}$

A previously healthy 71-year old woman came to our Movement Disorders Unit complaining of short, continuous, undulating involuntary movements in the right facial region at the cheekbone level only evident while looking at the mirror as she did not noticed them. There was no apparent triggering factor nor any associated symptoms. She became aware of them 10 days ago. She was questioned about family history, drug intake, trauma and recent infections, all of which were negative.

At examination brief repetitive rhythmic undulating localized contractions in the right facial region were evident (Video 1). There was no atrophy, weakness or sensory disturbances. The remainder of the neurological examination was normal.

Video I Brief muscle contractions in the right check.

A brain MRI showed only limited areas of focal gliosis on the corona radiata, periventricular white matter and cerebral white matter and a lacunae on the right lenticular capsular region. A magnetic resonance angiography was normal. Electromyography showed rhythmic discharges at $3 \mathrm{~Hz}$ consistent with myokymia in the levator labii superioris and oribuclaris oris muscles (Video 2).

Video 2 EMG recording sharing brief discharges consistent with myokymia.
Due to these findings, 5 units of onabotulinumtoxinA (Botox) were injected at the levator muscle of the upper lip on the right side, and myokymia completely disappeared in 48 hours.

Facial myokymia, most commonly involves the orbicularis oculi muscle, usually on the lower eyelid, it is frequently secondary to ipsilateral pontine tegmentum lesions. It is generally self-limited and lasts a few days although it can occasionally persist for various weeks or months. ${ }^{4}$ The pathogenesis is still unclear. Given the association of myokymia with a variety of lesion sites, its origin seems to occur at any level of the motor pathway from the motor neurons to the distal segments of the nerve terminal. Facial myokymia is believed to originate in the facial motor nucleus either by direct irritation, hyper-excitation, hyper-sensitivity to secondary denervation of a supranuclear process or motor-neural isolation of the facial nucleus of the internuncial neuron pool that mediates the sensory afference. ${ }^{3}$

In some cases it can be secondary to pontine tumors acoustic neuroma, metastatic disease or multiple sclerosis; vascular disorders, tuberculomas, syringobulbia, Guillain-Barré syndrome, multiple system atrophy (MSA), spinocerebellar ataxia type 3 (SCA3), cysticercosis, autosomal dominant striatonigral degeneration, or Bell's palsy ${ }^{5}$ and even autoimmune. In our patient myokymia was idiopathic.

For patients with focalized myokymia, botulinum toxin has been suggested as a useful treatment. Although there are very few reported cases in the literature in which this therapy has been administered and a case of facial myokymia and regional synkinesis has been described with a good response to carbamazepine. Our cases illustrates the usefulness of low doses of Onabotulinumtoxin A in the treatment of continuous idiopathic facial myokymia.

\section{Video statement}

Subject gave consent to be videoed for publication.

The patient signed consent to be filmed for publication on line.

\section{Acknowledgments}

None.

\section{Conflicts of interest}

None. 


\section{Funding}

None.

\section{References}

1. Banik R, Miller NR. Chronic myokymia limited to the eyelid is a benign condition. J Neuroophtalmol. 2004;24(4):290-292.

2. Gutmann L. Myokymia and neuromyotonia. $J$ Neurol. 2004;251(2):138-142.
3. Bathia R, Desai S. Isolated facial myokymia as a presenting feature of pontine neurocysticercosis. Mov Disord. 2008;23(1):135-137.

4. Banik R, Miller NR. Chronic myokymia limited to the eyelid is a benign condition. J Neuroophthalmol. 2004;24(4):290-292.

5. Jacobs L, Kaba S, Pullicino P. The lesion causing continuous facial myokymia in multiple sclerosis. Arch Neurol. 1994;51(11):1115-1119. 